\title{
Hadamard-type and Bullen-type inequalities for Lipschitzian functions via fractional integrals
}

\author{
İmdat İşcan*
}

(Communicated by Nihal YILMAZ ÖZGÜR)

\begin{abstract}
In this paper, the author establishes some Hadamard-type and Bullen-type inequalities for Lipschitzian functions via Riemann Liouville fractional integral.
\end{abstract}

Keywords: Lipschitzian functions; Hadamard inequality; Bullen inequality; Riemann-Liouville fractional integral.

AMS Subject Classification (2010): Primary: 26A51 ; Secondary: 26A33; 26D15.

*Corresponding author

\section{Introduction}

Following inequality is well known in the literature as Hermite-Hadamard's inequality:

Theorem 1.1. Let $f: I \subseteq \mathbb{R} \rightarrow \mathbb{R}$ be a convex function defined on the interval I of real numbers and $a, b \in I$ with $a<b$. The following double inequality holds

$$
f\left(\frac{a+b}{2}\right) \leq \frac{1}{b-a} \int_{a}^{b} f(x) d x \leq \frac{f(a)+f(b)}{2} .
$$

In [8], Tseng et al. established the following Hadamard-type inequality which refines the inequality (1.1).

Theorem 1.2. Suppose that $f:[a, b] \rightarrow \mathbb{R}$ is a convex function on $[a, b]$. Then we have the inequalities:

$$
\begin{aligned}
f\left(\frac{a+b}{2}\right) & \leq \frac{1}{2}\left[f\left(\frac{3 a+b}{4}\right)+f\left(\frac{a+3 b}{4}\right)\right] \\
& \leq \frac{1}{b-a} \int f(x) d x \\
& \leq \frac{1}{2}\left[f\left(\frac{a+b}{2}\right)+\frac{f(a)+f(b)}{2}\right] \leq \frac{f(a)+f(b)}{2} .
\end{aligned}
$$

The third inequality in (1.2) is known in the literature as Bullen's inequality.

In what follows we recall the following definition.

Definition 1.1. A function $f: I \subseteq \mathbb{R} \rightarrow \mathbb{R}$ is called an $M$-Lipschitzian function on the interval $I$ of real numbers with $M \geq 0$, if

$$
|f(x)-f(y)| \leq M|x-y|
$$

for all $x, y \in I$.

Received : 04-March-2015, Accepted : 22-February-2016 
For some recent results connected with Hermite-Hadamard type integral inequalities for Lipschitzian functions, see $[2-4,9,10]$.

In [9], Tseng et al. established some Hadamard-type and Bullen-type inequalities for Lipschitzian functions as follows.

Theorem 1.3. Let $I$ be an interval in $\mathbb{R}, a \leq A \leq B \leq b$ in $I, V=(1-\alpha) a+\alpha b, \alpha \in[0,1]$ and let $f: I \rightarrow \mathbb{R}$ be an $L$-Lipschitzian function with $L \geq 0$. Then we have the inequality

$$
\left|\alpha f(A)+(1-\alpha) f(B)-\frac{1}{b-a} \int_{a}^{b} f(x) d x\right| \leq \frac{L V_{\alpha}(A, B)}{b-a},
$$

where

$$
\begin{aligned}
& V_{\alpha}(A, B) \\
& \left\{\begin{array}{c}
(A-a)^{2}-(A-V)^{2}+(B-V)^{2}+(b-B)^{2}, \\
a \leq V \leq A \leq B \leq b, \\
(A-a)^{2}+(V-A)^{2}+(B-V)^{2}+(b-B)^{2}, \\
a \leq A \leq V \leq B \leq b, \\
(A-a)^{2}+(V-A)^{2}+(b-B)^{2}-(V-B)^{2}, \\
a \leq A \leq B \leq V \leq b
\end{array} .\right.
\end{aligned}
$$

Theorem 1.4. Let I be an interval in $\mathbb{R}, a \leq A \leq B \leq C \leq b$ in $I, V_{1}=(1-\alpha) a+\alpha b, V_{2}=\gamma a+(\alpha+\beta) b, \alpha, \beta, \gamma \in[0,1]$, $\alpha+\beta+\gamma=1$, and let $f: I \rightarrow \mathbb{R}$ be an $L$-Lipschitzian function with $L \geq 0$. Then we have the inequality

$$
\left|\alpha f(A)+\beta f(B)+\gamma f(C)-\frac{1}{b-a} \int_{a}^{b} f(x) d x\right| \leq \frac{L V_{\alpha, \beta, \gamma}(A, B, C)}{b-a},
$$

where $V_{\alpha, \beta, \gamma}$ is defined as in [9, Section 3].

We give some necessary definitions and mathematical preliminaries of fractional calculus theory which are used throughout this paper.

Definition 1.2. Let $f \in L[a, b]$. The Riemann-Liouville integrals $J_{a^{+}}^{\alpha} f$ and $J_{b^{-}}^{\alpha} f$ of order $\alpha>0$ with $a \geq 0$ are defined by

$$
J_{a^{+}}^{\alpha} f(x)=\frac{1}{\Gamma(\alpha)} \int_{a}^{x}(x-t)^{\alpha-1} f(t) d t, x>a
$$

and

$$
J_{b^{-}}^{\alpha} f(x)=\frac{1}{\Gamma(\alpha)} \int_{x}^{b}(t-x)^{\alpha-1} f(t) d t, x<b
$$

respectively, where $\Gamma(\alpha)$ is the Gamma function defined by $\Gamma(\alpha)=\int_{0}^{\infty} e^{-t} t^{\alpha-1} d t$ and $J_{a^{+}}^{0} f(x)=J_{b^{-}}^{0} f(x)=f(x)$ (see [5]).

In the case of $\alpha=1$, the fractional integral reduces to the classical integral. For some recent results connected with fractional integral inequalities, see $[1,6,7,11]$.

The aim of this paper is to establish some Hadamard-type and Bullen-type inequalities for Lipschitzian functions via Riemann-Liouville fractional integral. The results obtained herein is a generalization of the results obtained in Theorem 3 and Theorem 4 via fractional integrals. 


\section{Hadamard-type inequalities for Lipschitzian functions via fractional integrals}

Throughout this section, let $I$ be an interval in $\mathbb{R}, a \leq x \leq y \leq b$ in $I$ and let $f: I \rightarrow \mathbb{R}$ be an $M$-Lipschitzian function. In the next theorem, let $\lambda \in[0,1], V=(1-\lambda) a+\lambda b$, and $V_{\alpha, \lambda}, \alpha>0$, as follows:

(1) If $a \leq V \leq x \leq y \leq b$, then

$$
V_{\alpha, \lambda}(x, y)=(V-a)^{\alpha}\left[\frac{x-a}{\alpha}-\frac{V-a}{\alpha+1}\right]+\frac{2(b-y)^{\alpha+1}}{\alpha(\alpha+1)}+(b-V)^{\alpha}\left[\frac{b-V}{\alpha+1}-\frac{b-y}{\alpha}\right]
$$

(2) If $a \leq x \leq V \leq y \leq b$, then

$$
\begin{aligned}
V_{\alpha, \lambda}(x, y)= & \frac{2(x-a)^{\alpha+1}}{\alpha(\alpha+1)}+(V-a)^{\alpha}\left[\frac{V-a}{\alpha+1}-\frac{x-a}{\alpha}\right] \\
& +\frac{2(b-y)^{\alpha+1}}{\alpha(\alpha+1)}+(b-V)^{\alpha}\left[\frac{b-V}{\alpha+1}-\frac{b-y}{\alpha}\right] .
\end{aligned}
$$

(3) If $a \leq x \leq y \leq V \leq b$, then

$$
V_{\alpha, \lambda}(x, y)=\frac{2(x-a)^{\alpha+1}}{\alpha(\alpha+1)}+(V-a)^{\alpha}\left[\frac{V-a}{\alpha+1}-\frac{x-a}{\alpha}\right]+(b-V)^{\alpha}\left[\frac{b-y}{\alpha}-\frac{b-V}{\alpha+1}\right] .
$$

Theorem 2.1. Let $x, y, \alpha, \lambda, V, V_{\alpha, \lambda}$ and the function $f$ be defined as above. Then we have the inequality for fractional integrals

$$
\left|\lambda^{\alpha} f(x)+(1-\lambda)^{\alpha} f(y)-\frac{\Gamma(\alpha+1)}{(b-a)^{\alpha}}\left[J_{V-}^{\alpha} f(a)+J_{V+}^{\alpha} f(b)\right]\right| \leq \frac{\alpha M V_{\alpha, \lambda}(x, y)}{(b-a)^{\alpha}} .
$$

Proof. Using the hypothesis of $f$, we have the following inequality

$$
\begin{aligned}
& \left|\lambda^{\alpha} f(x)+(1-\lambda)^{\alpha} f(y)-\frac{\Gamma(\alpha+1)}{(b-a)^{\alpha}}\left[J_{V-f}^{\alpha} f(a)+J_{V+}^{\alpha} f(b)\right]\right| \\
= & \frac{\alpha}{(b-a)^{\alpha}}\left|\int_{a}^{V}[f(x)-f(t)](t-a)^{\alpha-1} d t+\int_{V}^{b}[f(y)-f(t)](b-t)^{\alpha-1} d t\right| \\
\leq & \frac{\alpha}{(b-a)^{\alpha}}\left[\int_{a}^{V}|f(x)-f(t)|(t-a)^{\alpha-1} d t+\int_{V}^{b}|f(y)-f(t)|(b-t)^{\alpha-1} d t\right] \\
\leq & \frac{\alpha M}{(b-a)^{\alpha}}\left[\int_{a}^{V}|x-t|(t-a)^{\alpha-1} d t+\int_{V}^{b}|y-t|(b-t)^{\alpha-1} d t\right] .
\end{aligned}
$$

Now using simple calculations, we obtain the following identities $\int_{a}^{V}|x-t|(t-a)^{\alpha-1} d t$ and $\int_{V}^{b}|y-t|(b-t)^{\alpha-1} d t$.

(1) If $a \leq V \leq x \leq y \leq b$, then

$$
\int_{a}^{V}|x-t|(t-a)^{\alpha-1} d t=(V-a)^{\alpha}\left[\frac{x-a}{\alpha}-\frac{V-a}{\alpha+1}\right]
$$

and

$$
\int_{V}^{b}|y-t|(b-t)^{\alpha-1} d t=\frac{2(b-y)^{\alpha+1}}{\alpha(\alpha+1)}+(b-V)^{\alpha}\left[\frac{b-V}{\alpha+1}-\frac{b-y}{\alpha}\right] .
$$

(2) If $a \leq x \leq V \leq y \leq b$, then

$$
\int_{a}^{V}|x-t|(t-a)^{\alpha-1} d t=\frac{2(x-a)^{\alpha+1}}{\alpha(\alpha+1)}+(V-a)^{\alpha}\left[\frac{V-a}{\alpha+1}-\frac{x-a}{\alpha}\right]
$$


and

$$
\int_{V}^{b}|y-t|(b-t)^{\alpha-1} d t=\frac{2(b-y)^{\alpha+1}}{\alpha(\alpha+1)}+(b-V)^{\alpha}\left[\frac{b-V}{\alpha+1}-\frac{b-y}{\alpha}\right] .
$$

(3) If $a \leq x \leq y \leq V \leq b$, then

$$
\int_{a}^{V}|x-t|(t-a)^{\alpha-1} d t=\frac{2(x-a)^{\alpha+1}}{\alpha(\alpha+1)}+(V-a)^{\alpha}\left[\frac{V-a}{\alpha+1}-\frac{x-a}{\alpha}\right]
$$

and

$$
\int_{V}^{b}|y-t|(b-t)^{\alpha-1} d t=(b-V)^{\alpha}\left[\frac{b-y}{\alpha}-\frac{b-V}{\alpha+1}\right] .
$$

Using the inequality (2.2) and the above identities $\int_{a}^{V}|x-t|(t-a)^{\alpha-1} d t$ and $\int_{V}^{b}|y-t|(b-t)^{\alpha-1} d t$, we derive the inequality (2.1). This completes the proof.

Under the assumptions of Theorem 2.1, we have the following corollaries and remarks:

Remark 2.1. In Theorem 2.1, if we take $\alpha=1$, then the inequality (2.1) reduces the inequality (1.3) in Theorem 1.3.

Corollary 2.1. 1. In Theorem 2.1, let $\delta \in\left[\frac{1}{2}, 1\right], x=\delta a+(1-\delta) b$ and $y=(1-\delta) a+\delta b$. Then, we have the inequality

$$
\begin{gathered}
\left|\lambda^{\alpha} f(\delta a+(1-\delta) b)+(1-\lambda)^{\alpha} f((1-\delta) a+\delta b)-\frac{\Gamma(\alpha+1)}{(b-a)^{\alpha}}\left[J_{V-}^{\alpha} f(a)+J_{V+}^{\alpha} f(b)\right]\right| \\
\leq \frac{M L(\alpha, \lambda, \delta)(b-a)}{\alpha+1}
\end{gathered}
$$

where

$$
\begin{gathered}
L(\alpha, \lambda, \delta) \\
=\left\{\begin{array}{c}
\lambda^{\alpha}[(1-\delta)(1+\alpha)-\lambda \alpha]+2(1-\delta)^{\alpha+1}+(1-\lambda)^{\alpha}[(1-\lambda) \alpha-(1-\delta)(1+\alpha)], \\
\lambda \leq 1-\delta \\
4(1-\delta)^{\alpha+1}+\lambda^{\alpha}[\lambda \alpha-(1-\delta)(1+\alpha)]+(1-\lambda)^{\alpha}[(1-\lambda) \alpha-(1-\delta)(1+\alpha)], \\
1-\delta \leq \lambda \leq \delta \\
2(1-\delta)^{\alpha+1}+\lambda^{\alpha}[\lambda \alpha-(1-\delta)(1+\alpha)]+(1-\lambda)^{\alpha}[(1-\delta)(1+\alpha)-(1-\lambda) \alpha], \\
\delta \leq \lambda .
\end{array}\right.
\end{gathered}
$$

2. In Theorem 2.1, if we take $x=y=V$, then we have the inequality

$$
\begin{aligned}
& \left|\left[\lambda^{\alpha}+(1-\lambda)^{\alpha}\right] f(x)-\frac{\Gamma(\alpha+1)}{(b-a)^{\alpha}}\left[J_{V-}^{\alpha} f(a)+J_{V+}^{\alpha} f(b)\right]\right| \\
\leq & M \frac{(x-a)^{\alpha+1}+(b-x)^{\alpha+1}}{(\alpha+1)(b-a)^{\alpha}} .
\end{aligned}
$$

Corollary 2.2. We have the following weighted Hadamard-type inequalities for Lipschitzian functions via Rieamnn-liouville fractional integrals

(1) In the inequality (2.3), if we take $\delta=1$, then we have

$$
\begin{aligned}
& \left|\lambda^{\alpha} f(a)+(1-\lambda)^{\alpha} f(b)-\frac{\Gamma(\alpha+1)}{(b-a)^{\alpha}}\left[J_{V-}^{\alpha} f(a)+J_{V+}^{\alpha} f(b)\right]\right| \\
\leq & \alpha M(b-a) \frac{\lambda^{\alpha+1}+(1-\lambda)^{\alpha+1}}{\alpha+1},
\end{aligned}
$$


in this inequality, specially if we choose $\lambda=\frac{x-a}{b-a}$ for $x \in[a, b]$, then

$$
\begin{aligned}
& \left|\frac{(x-a)^{\alpha} f(a)+(b-x)^{\alpha} f(b)}{(b-a)^{\alpha}}-\frac{\Gamma(\alpha+1)}{(b-a)^{\alpha}}\left[J_{x-}^{\alpha} f(a)+J_{x+}^{\alpha} f(b)\right]\right| \\
\leq & \alpha M \frac{(x-a)^{\alpha+1}+(b-x)^{\alpha+1}}{(\alpha+1)(b-a)^{\alpha}}
\end{aligned}
$$

(2) In the inequality (2.4), if we take $x=\delta a+(1-\delta) b, \delta \in[0,1]$, then

$$
\begin{aligned}
& \left|\left[\lambda^{\alpha}+(1-\lambda)^{\alpha}\right] f(\delta a+(1-\delta) b)-\frac{\Gamma(\alpha+1)}{(b-a)^{\alpha}}\left[J_{V-}^{\alpha} f(a)+J_{V+}^{\alpha} f(b)\right]\right| \\
\leq & M(b-a) \frac{\delta^{\alpha+1}+(1-\delta)^{\alpha+1}}{(\alpha+1)},
\end{aligned}
$$

in this inequality, specially if we choose $\lambda=\frac{1}{2}$, then

$$
\begin{aligned}
& \left|f(\delta a+(1-\delta) b)-\frac{2^{\alpha-1} \Gamma(\alpha+1)}{(b-a)^{\alpha}}\left[J_{\left(\frac{a+b}{2}\right)-}^{\alpha} f(a)+J_{\left(\frac{a+b}{2}\right)+}^{\alpha} f(b)\right]\right| \\
\leq & 2^{\alpha-1} M(b-a) \frac{\delta^{\alpha+1}+(1-\delta)^{\alpha+1}}{(\alpha+1)},
\end{aligned}
$$

(3) In the inequality (2.4), if we take $\lambda=\frac{1}{2}$ and $\delta=\frac{3}{4}$ then

$$
\begin{aligned}
& \left|\frac{1}{2}\left[f\left(\frac{3 a+b}{4}\right)+f\left(\frac{a+3 b}{4}\right)\right]-\frac{2^{\alpha-1} \Gamma(\alpha+1)}{(b-a)^{\alpha}}\left[J_{\left(\frac{a+b}{2}\right)-}^{\alpha} f(a)+J_{\left(\frac{a+b}{2}\right)+}^{\alpha} f(b)\right]\right| \\
\leq & M(b-a) \frac{1+2^{\alpha-1}(\alpha-1)}{2^{\alpha+1}(\alpha+1)}
\end{aligned}
$$

\section{Bullen -type inequalities for Lipschitzian functions via fractional integrals}

Throughout this section, let $I$ be an interval in $\mathbb{R}, a \leq x \leq y \leq z \leq b$ in $I$ and $f: I \rightarrow \mathbb{R}$ be an $M$-lipschitzian function. In the next theorem, let $\lambda+\eta+\mu=1, \lambda, \eta, \mu \in[0,1], V_{1}=(1-\lambda) a+\lambda b, V_{2}=\mu a+(\lambda+\eta) b$, and define $V_{\alpha, \lambda, \eta, \mu}, \alpha>0$, as follows:

1. If $V_{1} \leq V_{2} \leq x \leq y \leq z$ or $V_{1} \leq x \leq V_{2} \leq y \leq z$, then

$$
\begin{aligned}
V_{\alpha, \lambda, \eta, \mu}(x, y, z)= & \left(V_{1}-a\right)^{\alpha}\left[\frac{x-a}{\alpha}-\frac{V_{1}-a}{\alpha+1}\right]+\left(V_{2}-V_{1}\right)^{\alpha}\left[\frac{y-V_{2}}{\alpha}+\frac{V_{2}-V_{1}}{\alpha+1}\right] \\
& +\frac{2(b-z)^{\alpha+1}}{\alpha(\alpha+1)}+\left(b-V_{2}\right)^{\alpha}\left[\frac{b-V_{2}}{\alpha+1}-\frac{b-z}{\alpha}\right] .
\end{aligned}
$$

2. If $V_{1} \leq x \leq y \leq V_{2} \leq z$, then

$$
\begin{aligned}
V_{\alpha, \lambda, \eta, \mu}(x, y, z)= & \left(V_{1}-a\right)^{\alpha}\left[\frac{x-a}{\alpha}-\frac{V_{1}-a}{\alpha+1}\right]+\frac{2\left(V_{2}-y\right)^{\alpha+1}}{\alpha(\alpha+1)}+\left(V_{2}-V_{1}\right)^{\alpha}\left[\frac{V_{2}-V_{1}}{\alpha+1}-\frac{V_{2}-y}{\alpha}\right] \\
& +\frac{2(b-z)^{\alpha+1}}{\alpha(\alpha+1)}+\left(b-V_{2}\right)^{\alpha}\left[\frac{b-V_{2}}{\alpha+1}-\frac{b-z}{\alpha}\right] .
\end{aligned}
$$

3. If $V_{1} \leq x \leq y \leq z \leq V_{2}$, then

$$
\begin{aligned}
V_{\alpha, \lambda, \eta, \mu}(x, y, z)= & \left(V_{1}-a\right)^{\alpha}\left[\frac{x-a}{\alpha}-\frac{V_{1}-a}{\alpha+1}\right]+\frac{2\left(V_{2}-y\right)^{\alpha+1}}{\alpha(\alpha+1)} \\
& +\left(V_{2}-V_{1}\right)^{\alpha}\left[\frac{V_{2}-V_{1}}{\alpha+1}-\frac{V_{2}-y}{\alpha}\right]+\left(b-V_{2}\right)^{\alpha}\left[\frac{b-z}{\alpha}-\frac{b-V_{2}}{\alpha+1}\right] .
\end{aligned}
$$


4. If $x \leq V_{1} \leq V_{2} \leq y \leq z$, then

$$
\begin{aligned}
V_{\alpha, \lambda, \eta, \mu}(x, y, z)= & \frac{2(x-a)^{\alpha+1}}{\alpha(\alpha+1)}+\left(V_{1}-a\right)^{\alpha}\left[\frac{V_{1}-a}{\alpha+1}-\frac{x-a}{\alpha}\right]+\left(V_{2}-V_{1}\right)^{\alpha}\left[\frac{y-V_{2}}{\alpha}+\frac{V_{2}-V_{1}}{\alpha+1}\right] \\
& +\frac{2(b-z)^{\alpha+1}}{\alpha(\alpha+1)}+\left(b-V_{2}\right)^{\alpha}\left[\frac{b-V_{2}}{\alpha+1}-\frac{b-z}{\alpha}\right] .
\end{aligned}
$$

5. If $x \leq V_{1} \leq y \leq V_{2} \leq z$, then

$$
\begin{aligned}
V_{\alpha, \lambda, \eta, \mu}(x, y, z)= & \frac{2(x-a)^{\alpha+1}}{\alpha(\alpha+1)}+\left(V_{1}-a\right)^{\alpha}\left[\frac{V_{1}-a}{\alpha+1}-\frac{x-a}{\alpha}\right]+\frac{2\left(V_{2}-y\right)^{\alpha+1}}{\alpha(\alpha+1)} \\
& +\left(V_{2}-V_{1}\right)^{\alpha}\left[\frac{V_{2}-V_{1}}{\alpha+1}-\frac{V_{2}-y}{\alpha}\right]+\frac{2(b-z)^{\alpha+1}}{\alpha(\alpha+1)}+\left(b-V_{2}\right)^{\alpha}\left[\frac{b-V_{2}}{\alpha+1}-\frac{b-z}{\alpha}\right] .
\end{aligned}
$$

6. If $x \leq V_{1} \leq y \leq z \leq V_{2}$, then

$$
\begin{aligned}
V_{\alpha, \lambda, \eta, \mu}(x, y, z)= & \frac{2(x-a)^{\alpha+1}}{\alpha(\alpha+1)}+\left(V_{1}-a\right)^{\alpha}\left[\frac{V_{1}-a}{\alpha+1}-\frac{x-a}{\alpha}\right]+\frac{2\left(V_{2}-y\right)^{\alpha+1}}{\alpha(\alpha+1)} \\
& +\left(V_{2}-V_{1}\right)^{\alpha}\left[\frac{V_{2}-V_{1}}{\alpha+1}-\frac{V_{2}-y}{\alpha}\right]+\left(b-V_{2}\right)^{\alpha}\left[\frac{b-z}{\alpha}-\frac{b-V_{2}}{\alpha+1}\right] .
\end{aligned}
$$

7. If $x \leq y \leq V_{1} \leq V_{2} \leq z$, then

$$
\begin{aligned}
V_{\alpha, \lambda, \eta, \mu}(x, y, z)= & \frac{2(x-a)^{\alpha+1}}{\alpha(\alpha+1)}+\left(V_{1}-a\right)^{\alpha}\left[\frac{V_{1}-a}{\alpha+1}-\frac{x-a}{\alpha}\right]+\left(V_{2}-V_{1}\right)^{\alpha}\left[\frac{V_{2}-V_{1}}{\alpha+1}-\frac{V_{2}-y}{\alpha}\right] \\
& +\frac{2(b-z)^{\alpha+1}}{\alpha(\alpha+1)}+\left(b-V_{2}\right)^{\alpha}\left[\frac{b-V_{2}}{\alpha+1}-\frac{b-z}{\alpha}\right] .
\end{aligned}
$$

8. If $x \leq y \leq V_{1} \leq z \leq V_{2}$ or $x \leq y \leq z \leq V_{1} \leq V_{2}$, then

$$
\begin{aligned}
V_{\alpha, \lambda, \eta, \mu}(x, y, z)= & \frac{2(x-a)^{\alpha+1}}{\alpha(\alpha+1)}+\left(V_{1}-a\right)^{\alpha}\left[\frac{V_{1}-a}{\alpha+1}-\frac{x-a}{\alpha}\right] \\
& +\left(V_{2}-V_{1}\right)^{\alpha}\left[\frac{V_{2}-y}{\alpha}-\frac{V_{2}-V_{1}}{\alpha+1}\right]+\left(b-V_{2}\right)^{\alpha}\left[\frac{b-z}{\alpha}-\frac{b-V_{2}}{\alpha+1}\right] .
\end{aligned}
$$

Theorem 3.1. Let $x, y, z, \lambda, \eta, \mu, V_{1}, V_{2}, V_{\alpha, \lambda, \eta, \mu}$ and the function $f$ be defined as above. Then we have the inequality

$$
\begin{aligned}
\mid \lambda^{\alpha} f(x)+\eta^{\alpha} f(y)+\mu^{\alpha} f(z) & -\frac{\Gamma(\alpha+1)}{(b-a)^{\alpha}}\left[J_{V_{1}-}^{\alpha} f(a)+J_{V_{1}+}^{\alpha} f\left(V_{2}\right)+J_{V_{2}+}^{\alpha} f(b)\right] \mid \\
\leq & \frac{\alpha M V_{\alpha, \lambda, \eta, \mu}(x, y, z)}{(b-a)^{\alpha}}
\end{aligned}
$$

Proof. Using the hypothesis of $f$, we have the inequality

$$
\begin{gathered}
\left|\lambda^{\alpha} f(x)+\eta^{\alpha} f(y)+\mu^{\alpha} f(z)-\frac{\Gamma(\alpha+1)}{(b-a)^{\alpha}}\left[J_{V_{1}-}^{\alpha} f(a)+J_{V_{1}+}^{\alpha} f\left(V_{2}\right)+J_{V_{2}+}^{\alpha} f(b)\right]\right| \\
\left.=\frac{\alpha}{(b-a)^{\alpha}} \mid \int_{a}^{V_{1}}[f(x)-f(t)](t-a)^{\alpha-1} d t+\int_{V_{1}}^{V_{2}}[f(y)-f(t)]\left(V_{2}-t\right)^{\alpha-1} d t+\int_{V_{2}}^{b}[f(z)-f(t)](b-t)^{\alpha-1} d t\right] \\
\leq \frac{\alpha}{(b-a)^{\alpha}}\left[\int_{a}^{V_{1}}|f(x)-f(t)|(t-a)^{\alpha-1} d t+\int_{V_{1}}^{V_{2}}|f(y)-f(t)|\left(V_{2}-t\right)^{\alpha-1} d t+\int_{V_{2}}^{b}|f(z)-f(t)|(b-t)^{\alpha-1} d t\right]
\end{gathered}
$$




$$
\leq \frac{\alpha M}{(b-a)^{\alpha}}\left[\int_{a}^{V_{1}}|x-t|(t-a)^{\alpha-1} d t+\int_{V_{1}}^{V_{2}}|y-t|\left(V_{2}-t\right)^{\alpha-1} d t+\int_{V_{2}}^{b}|z-t|(b-t)^{\alpha-1} d t\right] .
$$

Now, using simple calculations, we obtain the following identities $\int_{a}^{V_{1}}|x-t|(t-a)^{\alpha-1} d t, \int_{V_{1}}^{V_{2}}|y-t|\left(V_{2}-t\right)^{\alpha-1} d t$ and $\int_{V_{2}}^{b}|z-t|(b-t)^{\alpha-1} d t$.

1. If $V_{1} \leq V_{2} \leq x \leq y \leq z$ or $V_{1} \leq x \leq V_{2} \leq y \leq z$, then we have

$$
\begin{aligned}
\int_{a}^{V_{1}}|x-t|(t-a)^{\alpha-1} d t & =\left(V_{1}-a\right)^{\alpha}\left[\frac{x-a}{\alpha}-\frac{V_{1}-a}{\alpha+1}\right], \\
\int_{V_{1}}^{V_{2}}|y-t|\left(V_{2}-t\right)^{\alpha-1} d t & =\left(V_{2}-V_{1}\right)^{\alpha}\left[\frac{y-V_{2}}{\alpha}+\frac{V_{2}-V_{1}}{\alpha+1}\right]
\end{aligned}
$$

and

$$
\int_{V_{2}}^{b}|z-t|(b-t)^{\alpha-1} d t=\frac{2(b-z)^{\alpha+1}}{\alpha(\alpha+1)}+\left(b-V_{2}\right)^{\alpha}\left[\frac{b-V_{2}}{\alpha+1}-\frac{b-z}{\alpha}\right] .
$$

2. If $V_{1} \leq x \leq y \leq V_{2} \leq z$, then we have

$$
\begin{aligned}
\int_{a}^{V_{1}}|x-t|(t-a)^{\alpha-1} d t & =\left(V_{1}-a\right)^{\alpha}\left[\frac{x-a}{\alpha}-\frac{V_{1}-a}{\alpha+1}\right] \\
\int_{V_{1}}^{V_{2}}|y-t|\left(V_{2}-t\right)^{\alpha-1} d t & =\frac{2\left(V_{2}-y\right)^{\alpha+1}}{\alpha(\alpha+1)}+\left(V_{2}-V_{1}\right)^{\alpha}\left[\frac{V_{2}-V_{1}}{\alpha+1}-\frac{V_{2}-y}{\alpha}\right]
\end{aligned}
$$

and

$$
\int_{V_{2}}^{b}|z-t|(b-t)^{\alpha-1} d t=\frac{2(b-z)^{\alpha+1}}{\alpha(\alpha+1)}+\left(b-V_{2}\right)^{\alpha}\left[\frac{b-V_{2}}{\alpha+1}-\frac{b-z}{\alpha}\right] .
$$

3. If $V_{1} \leq x \leq y \leq z \leq V_{2}$, then we have

$$
\begin{aligned}
\int_{a}^{V_{1}}|x-t|(t-a)^{\alpha-1} d t & =\left(V_{1}-a\right)^{\alpha}\left[\frac{x-a}{\alpha}-\frac{V_{1}-a}{\alpha+1}\right] \\
\int_{V_{1}}^{V_{2}}|y-t|\left(V_{2}-t\right)^{\alpha-1} d t & =\frac{2\left(V_{2}-y\right)^{\alpha+1}}{\alpha(\alpha+1)}+\left(V_{2}-V_{1}\right)^{\alpha}\left[\frac{V_{2}-V_{1}}{\alpha+1}-\frac{V_{2}-y}{\alpha}\right]
\end{aligned}
$$

and

$$
\int_{V_{2}}^{b}|z-t|(b-t)^{\alpha-1} d t=\left(b-V_{2}\right)^{\alpha}\left[\frac{b-z}{\alpha}-\frac{b-V_{2}}{\alpha+1}\right] .
$$

4. If $x \leq V_{1} \leq V_{2} \leq y \leq z$, then we have

$$
\begin{aligned}
\int_{a}^{V_{1}}|x-t|(t-a)^{\alpha-1} d t & =\frac{2(x-a)^{\alpha+1}}{\alpha(\alpha+1)}+\left(V_{1}-a\right)^{\alpha}\left[\frac{V_{1}-a}{\alpha+1}-\frac{x-a}{\alpha}\right], \\
\int_{V_{1}}^{V_{2}}|y-t|\left(V_{2}-t\right)^{\alpha-1} d t & =\left(V_{2}-V_{1}\right)^{\alpha}\left[\frac{y-V_{2}}{\alpha}+\frac{V_{2}-V_{1}}{\alpha+1}\right],
\end{aligned}
$$


and

$$
\int_{V_{2}}^{b}|z-t|(b-t)^{\alpha-1} d t=\frac{2(b-z)^{\alpha+1}}{\alpha(\alpha+1)}+\left(b-V_{2}\right)^{\alpha}\left[\frac{b-V_{2}}{\alpha+1}-\frac{b-z}{\alpha}\right] .
$$

5. If $x \leq V_{1} \leq y \leq V_{2} \leq z$, then we have

$$
\begin{aligned}
& \int_{a}^{V_{1}}|x-t|(t-a)^{\alpha-1} d t=\frac{2(x-a)^{\alpha+1}}{\alpha(\alpha+1)}+\left(V_{1}-a\right)^{\alpha}\left[\frac{V_{1}-a}{\alpha+1}-\frac{x-a}{\alpha}\right] \\
& \int_{V_{1}}^{V_{2}}|y-t|\left(V_{2}-t\right)^{\alpha-1} d t=\frac{2\left(V_{2}-y\right)^{\alpha+1}}{\alpha(\alpha+1)}+\left(V_{2}-V_{1}\right)^{\alpha}\left[\frac{V_{2}-V_{1}}{\alpha+1}-\frac{V_{2}-y}{\alpha}\right]
\end{aligned}
$$

and

$$
\int_{V_{2}}^{b}|z-t|(b-t)^{\alpha-1} d t=\frac{2(b-z)^{\alpha+1}}{\alpha(\alpha+1)}+\left(b-V_{2}\right)^{\alpha}\left[\frac{b-V_{2}}{\alpha+1}-\frac{b-z}{\alpha}\right] .
$$

6. If $x \leq V_{1} \leq y \leq z \leq V_{2}$, then we have

$$
\begin{aligned}
\int_{a}^{V_{1}}|x-t|(t-a)^{\alpha-1} d t & =\frac{2(x-a)^{\alpha+1}}{\alpha(\alpha+1)}+\left(V_{1}-a\right)^{\alpha}\left[\frac{V_{1}-a}{\alpha+1}-\frac{x-a}{\alpha}\right] \\
\int_{V_{1}}^{V_{2}}|y-t|\left(V_{2}-t\right)^{\alpha-1} d t & =\frac{2\left(V_{2}-y\right)^{\alpha+1}}{\alpha(\alpha+1)}+\left(V_{2}-V_{1}\right)^{\alpha}\left[\frac{V_{2}-V_{1}}{\alpha+1}-\frac{V_{2}-y}{\alpha}\right]
\end{aligned}
$$

and

$$
\int_{V_{2}}^{b}|z-t|(b-t)^{\alpha-1} d t=\left(b-V_{2}\right)^{\alpha}\left[\frac{b-z}{\alpha}-\frac{b-V_{2}}{\alpha+1}\right] .
$$

7. If $x \leq y \leq V_{1} \leq V_{2} \leq z$, then we have

$$
\begin{aligned}
\int_{a}^{V_{1}}|x-t|(t-a)^{\alpha-1} d t & =\frac{2(x-a)^{\alpha+1}}{\alpha(\alpha+1)}+\left(V_{1}-a\right)^{\alpha}\left[\frac{V_{1}-a}{\alpha+1}-\frac{x-a}{\alpha}\right], \\
\int_{V_{1}}^{V_{2}}|y-t|\left(V_{2}-t\right)^{\alpha-1} d t & =\left(V_{2}-V_{1}\right)^{\alpha}\left[\frac{V_{2}-V_{1}}{\alpha+1}-\frac{V_{2}-y}{\alpha}\right]
\end{aligned}
$$

and

$$
\int_{V_{2}}^{b}|z-t|(b-t)^{\alpha-1} d t=\frac{2(b-z)^{\alpha+1}}{\alpha(\alpha+1)}+\left(b-V_{2}\right)^{\alpha}\left[\frac{b-V_{2}}{\alpha+1}-\frac{b-z}{\alpha}\right] .
$$

8. If $x \leq y \leq V_{1} \leq z \leq V_{2}$ or $x \leq y \leq z \leq V_{1} \leq V_{2}$, then we have

$$
\begin{aligned}
\int_{a}^{V_{1}}|x-t|(t-a)^{\alpha-1} d t & =\frac{2(x-a)^{\alpha+1}}{\alpha(\alpha+1)}+\left(V_{1}-a\right)^{\alpha}\left[\frac{V_{1}-a}{\alpha+1}-\frac{x-a}{\alpha}\right], \\
\int_{V_{1}}^{V_{2}}|y-t|\left(V_{2}-t\right)^{\alpha-1} d t & =\left(V_{2}-V_{1}\right)^{\alpha}\left[\frac{V_{2}-y}{\alpha}-\frac{V_{2}-V_{1}}{\alpha+1}\right],
\end{aligned}
$$


and

$$
\int_{V_{2}}^{b}|z-t|(b-t)^{\alpha-1} d t=\left(b-V_{2}\right)^{\alpha}\left[\frac{b-z}{\alpha}-\frac{b-V_{2}}{\alpha+1}\right] .
$$

Using the inequality (3.2) and the above identities $\int_{a}^{V_{1}}|x-t|(t-a)^{\alpha-1} d t, \int_{V_{1}}^{V_{2}}|y-t|\left(V_{2}-t\right)^{\alpha-1} d t$ and $\int_{V_{2}}^{b}|z-t|(b-t)^{\alpha-1} d t$, we derive the inequality (3.1). This completes the proof.

Under the assumptions of Theorem 3.1, we have the following corollaries and remarks:

Remark 3.1. In Theorem 3.1, if we take $\alpha=1$, then then the inequality (3.1) reduces the inequality (1.4) in Theorem 1.4 .

Corollary 3.1. In Theorem 3.1, let $\delta \in\left[\frac{1}{2}, 1\right], x=\delta a+(1-\delta) b, y=\frac{a+b}{2}$ and $z=(1-\delta) a+\delta b$. Then, we have the inequality

$$
\begin{gathered}
\left|\lambda^{\alpha} f(\delta a+(1-\delta) b)+\eta^{\alpha} f\left(\frac{a+b}{2}\right)+\mu^{\alpha} f((1-\delta) a+\delta b)-\frac{\Gamma(\alpha+1)}{(b-a)^{\alpha}}\left[J_{V_{1}-}^{\alpha} f(a)+J_{V_{1}+}^{\alpha} f\left(V_{2}\right)+J_{V_{2}+}^{\alpha} f(b)\right]\right| \\
\leq \frac{M N(\alpha, \lambda, \eta, \delta)(b-a)}{\alpha+1}
\end{gathered}
$$

where $N(\alpha, \lambda, \eta, \delta)$ is defined as follows:

1. If $\lambda+\eta \leq 1-\delta$ or $\lambda \leq 1-\delta \leq \lambda+\eta \leq \frac{1}{2}$, then

$$
\begin{aligned}
N(\alpha, \lambda, \eta, \delta)= & \lambda^{\alpha}[(1-\delta)(\alpha+1)-\alpha \lambda]+\eta^{\alpha}\left[\left(\frac{1}{2}-\lambda-\eta\right)(\alpha+1)+\alpha \eta\right] \\
& +2(1-\delta)^{\alpha+1}+(1-\lambda-\eta)^{\alpha}[\alpha(1-\lambda-\eta)-(\alpha+1)(1-\delta)] .
\end{aligned}
$$

2. If $\lambda \leq 1-\delta \leq \frac{1}{2} \leq \lambda+\eta \leq \delta$, then

$$
\begin{aligned}
N(\alpha, \lambda, \eta, \delta)= & \lambda^{\alpha}[(1-\delta)(\alpha+1)-\alpha \lambda]+2\left(\lambda+\eta-\frac{1}{2}\right)^{\alpha+1}+\eta^{\alpha}\left[\alpha \eta-(\alpha+1)\left(\lambda+\eta-\frac{1}{2}\right)\right] \\
& +2(1-\delta)^{\alpha+1}+(1-\lambda-\eta)^{\alpha}[\alpha(1-\lambda-\eta)-(\alpha+1)(1-\delta)] .
\end{aligned}
$$

3. If $\lambda \leq 1-\delta \leq \frac{1}{2} \leq \delta \leq \lambda+\eta$, then

$$
\begin{aligned}
N(\alpha, \lambda, \eta, \delta)= & \lambda^{\alpha}[(1-\delta)(\alpha+1)-\alpha \lambda]+2\left(\lambda+\eta-\frac{1}{2}\right)^{\alpha+1} \\
& +\eta^{\alpha}\left[\alpha \eta-(\alpha+1)\left(\lambda+\eta-\frac{1}{2}\right)\right]+(1-\lambda-\eta)^{\alpha}[(\alpha+1)(1-\delta)-\alpha(1-\lambda-\eta)] .
\end{aligned}
$$

4. If $1-\delta \leq \lambda \leq \lambda+\eta \leq \frac{1}{2}$, then

$$
\begin{aligned}
N(\alpha, \lambda, \eta, \delta)= & 4(1-\delta)^{\alpha+1}+\lambda^{\alpha}[\alpha \lambda-(1-\delta)(\alpha+1)]+\eta^{\alpha}\left[\alpha \eta+(\alpha+1)\left(\frac{1}{2}-\lambda-\eta\right)\right] \\
& +(1-\lambda-\eta)^{\alpha}[\alpha(1-\lambda-\eta)-(\alpha+1)(1-\delta)] .
\end{aligned}
$$

5. If $1-\delta \leq \lambda \leq \frac{1}{2} \leq \lambda+\eta \leq \delta$, then

$$
\begin{aligned}
N(\alpha, \lambda, \eta, \delta)= & 4(1-\delta)^{\alpha+1}+\lambda^{\alpha}[\alpha \lambda-(1-\delta)(\alpha+1)]+2\left(\lambda+\eta-\frac{1}{2}\right)^{\alpha+1} \\
& +\eta^{\alpha}\left[\alpha \eta-(\alpha+1)\left(\lambda+\eta-\frac{1}{2}\right)\right]+(1-\lambda-\eta)^{\alpha}[\alpha(1-\lambda-\eta)-(\alpha+1)(1-\delta)] .
\end{aligned}
$$


6. If $1-\delta \leq \lambda \leq \frac{1}{2} \leq \delta \leq \lambda+\eta$, then

$$
\begin{aligned}
N(\alpha, \lambda, \eta, \delta)= & 2(1-\delta)^{\alpha+1}+\lambda^{\alpha}[\alpha \lambda-(1-\delta)(\alpha+1)]+2\left(\lambda+\eta-\frac{1}{2}\right)^{\alpha+1} \\
& +\eta^{\alpha}\left[\alpha \eta-(\alpha+1)\left(\lambda+\eta-\frac{1}{2}\right)\right]+(1-\lambda-\eta)^{\alpha}[(\alpha+1)(1-\delta)-\alpha(1-\lambda-\eta)] .
\end{aligned}
$$

7. If $\frac{1}{2} \leq \lambda \leq \lambda+\eta \leq \delta$, then

$$
\begin{aligned}
N(\alpha, \lambda, \eta, \delta)= & 4(1-\delta)^{\alpha+1}+\lambda^{\alpha}[\alpha \lambda-(1-\delta)(\alpha+1)]+\eta^{\alpha}\left[\alpha \eta-(\alpha+1)\left(\lambda+\eta-\frac{1}{2}\right)\right] \\
& +(1-\lambda-\eta)^{\alpha}[\alpha(1-\lambda-\eta)-(\alpha+1)(1-\delta)] .
\end{aligned}
$$

8. If $\frac{1}{2} \leq \lambda \leq \delta \leq \lambda+\eta$ or $\delta \leq \lambda$, then

$$
\begin{aligned}
N(\alpha, \lambda, \eta, \delta)= & 2(1-\delta)^{\alpha+1}+\lambda^{\alpha}[\alpha \lambda-(1-\delta)(\alpha+1)] \\
& +\eta^{\alpha}\left[(\alpha+1)\left(\lambda+\eta-\frac{1}{2}\right)-\alpha \eta\right]+(1-\lambda-\eta)^{\alpha}[(\alpha+1)(1-\delta)-\alpha(1-\lambda-\eta)] .
\end{aligned}
$$

Corollary 3.2. In Corollary 3.1, if we take $\delta=1, \lambda=\mu=\frac{\theta}{2}$ and $\eta=1-\theta$ with $\theta \in[0,1]$, then we have the following weighted Bullen-type inequality for M-Lipschitzian functions via fractional integrals

$$
\begin{gathered}
\mid\left(\frac{\theta}{2}\right)^{\alpha}\left(f(a)+f(b)+(1-\theta)^{\alpha} f\left(\frac{a+b}{2}\right)+-\frac{\Gamma(\alpha+1)}{(b-a)^{\alpha}}\left[J_{V_{1}-}^{\alpha} f(a)+J_{V_{1}+}^{\alpha} f\left(V_{2}\right)+J_{V_{2}+}^{\alpha} f(b)\right] \mid\right. \\
\leq \frac{M\left[2 \alpha\left(\frac{\theta}{2}\right)^{\alpha+1}+(1-\theta)^{\alpha+1} \frac{\alpha-1}{2}+2\left(\frac{1-\theta}{2}\right)^{\alpha+1}\right](b-a)}{\alpha+1} .
\end{gathered}
$$

Remark 3.2. In the inequality (3.3), if we take $\theta=\frac{1}{2}$, then the inequality (3.3) reduces to the following Bullen-type inequality for $M$-Lipschitzian functions via fractional integrals

$$
\begin{aligned}
\mid \frac{1}{2}\left[\frac{f(a)+f(b)}{2}+f\left(\frac{a+b}{2}\right)\right] & -\frac{2^{\alpha-1} \Gamma(\alpha+1)}{(b-a)^{\alpha}}\left[J_{\left(\frac{3 a+b}{4}\right)-}^{\alpha} f(a)+J_{\left(\frac{3 a+b}{4}\right)+}^{\alpha} f\left(\frac{a+3 b}{4}\right)+J_{\left(\frac{a+3 b}{4}\right)+}^{\alpha} f(b)\right] \mid \\
& \leq \frac{M(b-a)}{2^{\alpha+2}(\alpha+1)}\left[\alpha+1+2^{\alpha-1}(\alpha-1)\right] .
\end{aligned}
$$

Remark 3.3. In the inequality (3.3), if we take $\theta=\frac{1}{3}$, then the inequality (3.3) reduces to the following Simpson-type inequality for $M$-Lipschitzian functions via fractional integrals

$$
\begin{aligned}
\mid \frac{1}{6}\left[f(a)+4 f\left(\frac{a+b}{2}\right)+f(b)\right] & -\frac{6^{\alpha-1} \Gamma(\alpha+1)}{(b-a)^{\alpha}}\left[J_{\left(\frac{5 a+b}{6}\right)-}^{\alpha} f(a)+J_{\left(\frac{5 a+b}{6}\right)+}^{\alpha} f\left(\frac{a+5 b}{6}\right)+J_{\left(\frac{a+5 b}{6}\right)+}^{\alpha} f(b)\right] \mid \\
\leq & \frac{M(b-a)}{18(\alpha+1)}\left[\alpha+2^{2 \alpha}(\alpha-1) 3+2^{\alpha+1}\right] .
\end{aligned}
$$

\section{References}

[1] Dahmani, Z., On Minkowski and Hermite-Hadamard integral inequalities via fractional via fractional integration. Ann. Funct. Anal. 1 (2010), no. 1, 51-58.

[2] Dragomir, S.S., Cho, Y.J. and Kim, S.S., Inequalities of Hadamard's Type for Lipschitzian Mappings and Their Applications. J. Math. Anal. Appl. 245 (2000), 489-501.

[3] Hwang, S.-R., Hsu, K.-C. and Tseng, K.-L., Hadamard-type inequalities for Lipschitzian functions in one and two variables with applications. J. Math. Anal. Appl. 405 (2013), 546-554. 
[4] İşcan, İ., New general integral inequalities for Lipschitzian functions via Hadamard fractional integrals. Int. J. Anal. 2014 (2014), Article ID 353924, 8 pages.

[5] Samko, S.G., Kilbas, A.A. and Marichev, O.I., Fractional Integrals and Derivatives Theory and Application. Gordan and Breach Science, New York, 1993.

[6] Sarıkaya, M.Z. and Ogunmez, H., On new inequalities via Riemann-Liouville fractional integration. Abstr. Appl. Anal. 2012 (2012), Article ID 428983, 10 pages.

[7] Sarıkaya, M.Z., Set, E., Yaldız, H. and Başak, N., Hermite-Hadamard's inequalities for fractional integrals and related fractional inequalities. Math. Comput. Modelling 57 (2013), 2403-2407.

[8] Tseng, K.-L., Hwang, S.-R. and Dragomir, S.S., Fejér-type inequalities (1). J. Inequal. Appl. 2010 (2010), Article ID 531976,7 pages.

[9] Tseng, K.-L., Hwang, S.-R. and Hsu, K.-C., Hadamard-type and Bullen-type inequalities for Lipschitzian functions and their applications. Comput. Math. Appl. 64 (2012), no. 4, 651-660.

[10] Yang, G.-S. and Tseng, K.-L., Inequalities of Hadamard's Type for Lipschitzian Mappings. J. Math. Anal. Appl. 260 (2001), no. 1, 230-238.

[11] Zhu, C., Fečkan, M. and Wang, J., Fractional integral inequalities for differentiable convex mappings and applications to special means and a midpoint formula. J. Appl. Math. Stat. Inform. 8 (2012), no. 2, 21-28.

\section{Affiliations}

İMDAT İşCAN

ADdRESS: Giresun University, Dept. of Mathematics, Giresun-Turkey.

E-MAIL: imdat.iscan@giresun.edu.tr 